\title{
A set for High-level comprehensive integrated crosstalk and delay estimation models
}

\author{
Nurbiya Yadikar ${ }^{1}$, Shun $\mathrm{Li}^{1}$, Hornisa Mamat ${ }^{1}$, Mutallip Mamut ${ }^{2}$, Kurban Ubul $^{1, \text { a }}$ \\ ${ }^{1}$ School of Information Science and Engineering, Xinjiang University, Urumqi, 830046, China \\ ${ }^{2}$ Library of , Xinjiang University, Urumqi, 830046, China \\ a corresponding author mail: kurbanu@xju.edu.cn
}

Keywords: multilayer interconnected system, crosstalk, interconnect, estimation model

\begin{abstract}
With the deepening of research, increasingly signal distortion and logic errors are no longer caused by the interconnection of line,in the multilayer interconnected system, the coupling and delay between the signals have a great influence on the performance of the system, Therefore, the VLSI circuit design must be accurate and celerity integrated crosstalk estimation. In this paper, we propose a new approximate crosstalk formula which can be used for high-level synthesis, and a new simple approximate crosstalk formula is proposed. It is based on the analysis of lumped parameters and giving the results of distribution parameters. The corresponding approximate formulas can be used to estimate the interconnect crosstalk quickly and accurately in the design planning. The results are not exactly the same as before. Compared with the test data, the new estimation model is superior to the previous results in both date accuracy and operation speed.
\end{abstract}

\section{Introduction}

With the development of science and technology, increasingly signal distortion and logic errors are no longer caused by the interconnection of line.The importance of this issue is being paid increasingly attention. The frequency of the system is a complex multilayer interconnection system, the VLSI circuit design must be accurate and celerity integrated crosstalk estimation.

Crosstalk noise must be analyzed in the early stage of design to develop reliable VLSI interconnects. The existing crosstalk model [1] has been applied to the analysis of distributed RC networks, and giving the strict solution of the step response. Subsequently, the RC model of the approximate lumped parameter is proposed in the crosstalk model [2], and obtain a better approximate simulation scheme. In the case of high frequency and high speed,it pointed out [3] that the inductance effect can not be ignored.M. Becer et al. derive a model [4] to accurately estimate the delay and crosstalk of two coupled interconnect lines switching simultaneously.V. Maheshwari et al. Presnt a model [6] accurately estimates the performance parameters of driver interconnect load system. They present [7] a closed form crosstalk noise estimation technique to coupled RLC interconnects based on coupled transmission line theory and Fourier series analysis.Capacitance approximation of distributed RC lines using 2- $\pi$ model [9].

In this paper, we analyze the impact of finite resistance in coupled RLC interconnects. The nonlinear behavior of the driver (CMOS inverter) is captured well in the proposed model. We introduce a novel technique to model CMOS inverter, namely MRLM (Multiple Ramp Library Model). It replaces the nonlinear driver into its equivalent RC model. We propose a new approximate crosstalk formula which can be used for high-level synthesis, and a new simple approximate crosstalk formula is proposed. The simulation results prove the correctness of the formula. The results are not exactly the same as before. Compared with the test data, the new model is superior to the previous results in both date accuracy and operation speed.

\section{Integrated crosstalk and delay estimation models under RC}

Due to the shielding effect of adjacent interconnects line to the far line, just consider the crosstalk between adjacent interconnects. Firstly, considering the effect of ignoring inductance, 
giving the linear hypothesis of CMOS circuit device, that is, the drive port has a driving resistor, and a load capacitance is arranged at the load port; the long interconnects line are divided into a number of short segments,I use mixed-models for each line segment, with the number of segments tends to infinity, the distribution parameters conclusion can be obtained by using the lumped circuit model. The circuit model is shown in Figure 1.Call these two interconnects $\operatorname{lineV}_{1,0}, V_{2,0}$ were line 1 and line two.

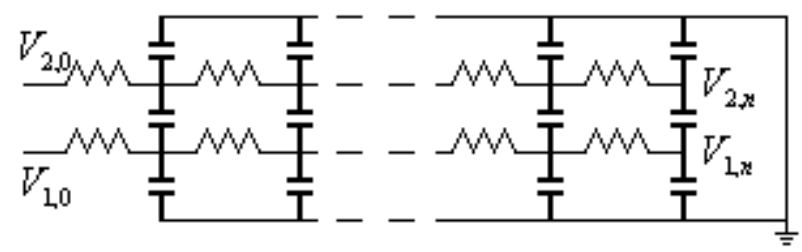

Figure 1 Interconnect diagram with mixed-model $M$ express resistance $\mathrm{R}$ けト capacitance $\mathrm{C}$ In the discussion of crosstalk problems, the simulation model of crosstalk response and the peak value of crosstalk estimation are given under consider the condition of inductance effect. Firstly, giving the equation of the circuit model and the answer, and the worst case crosstalk response, then analyzing the crosstalk response and giving the analysis results under certain conditions. Last, the formula of the peak value of crosstalk and the simple approximate formula are given. In order to establish the estimation model equation under RC condition, equivalent Figure 1 to figure 2,obtaining a cascade of similar trees. This response corresponds to line one for the adjacent interconnects in parallel,line two is the interference of the interconnect line.

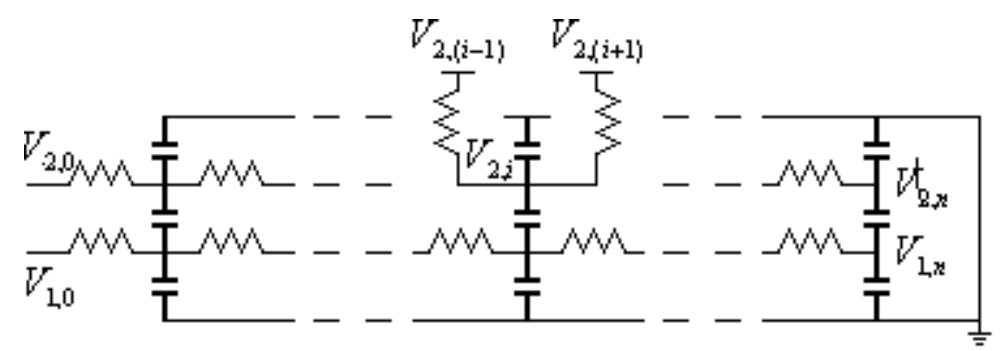

Figure 2 Schematic diagram of interconnect tree model $M$ express resistance $\mathrm{R}$ $\dashv \vdash$ capacitance C

\section{Crosstalk estimation and simulation analysis}

Matlab is both a powerful computational environment and a programming language that easily handles matrix and complex arithmetic.In this paper, the simulation environment is Matlab.

In the process of studying crosstalk,the worst case crosstalk impulse response of the $S$ domain is expanded to [5]:

$G(s)=m_{1} s+m_{2} s^{2}+m_{3} s^{3}+\cdots+m_{n} s^{n}+\cdots$

After calculation can obtain the Expression of $S$ domain coefficients about :

$$
\begin{aligned}
m_{1} & =\left(R_{S}+\frac{1}{2} R\right) C_{o x} \\
m_{2} & =-\left[\left(R_{S}^{2}+\frac{3}{4} R_{S} R+\frac{3}{22} R^{2}\right)\left(2 C+\frac{4}{3} C_{o x}\right)+\left(2 R_{S}^{2}+\frac{4}{7} R_{S} R+\frac{5}{7} R^{2}\right) C_{L}\right] C_{o x} \\
m_{3}= & {\left[\left(R_{S}^{3}+\frac{8}{7} R_{S}^{2} R+\frac{62}{123} R_{S} R^{2}+\frac{62}{861} R^{3}\right)\left(2 C^{2}+\frac{8}{3} C C_{o x}+\frac{4}{3} C_{o x}^{2}\right)+\left(2 R_{S}^{3}+\frac{13}{4} R_{S}^{2} R+\frac{63}{25} R_{S} R^{2}+\frac{63}{122} R^{3}\right)\left(C+\frac{4}{3} C_{o x}\right) C_{L}\right.} \\
& \left.+\left(2 R_{S}^{3}+\frac{14}{3} R_{S}^{2} R+\frac{16}{5} R_{S} R^{2}+\frac{5}{2} R^{3}\right) C_{L}^{2}\right] C_{o x}
\end{aligned}
$$


A two order system is used to approximate the shock response $\mathrm{H}(\mathrm{s})$ :

$$
\begin{gathered}
G(s)=m_{1} s+m_{2} s^{2}+m_{3} s^{3}+\cdots \approx \frac{m_{1} s}{1+\frac{-m_{2}}{m_{1}} s+\frac{-m_{3} m_{1}+m_{2}^{2}}{m_{1}^{2}} s^{2}}=\frac{a s}{1+\frac{2 \xi}{\omega} s+\frac{1}{\omega^{2}} s^{2}} \\
a=m_{1}, \omega=\sqrt{\frac{m_{1}^{2}}{m_{2}^{2}-m_{3} m_{1}}}, \xi=\frac{-m_{2}}{2 m_{1}} \omega
\end{gathered}
$$

Under the range condition that the step input is $\mathrm{Vk}$,Crosstalk output of the $\mathrm{S}$ domain is transformed into:

$$
V(s)=\frac{V_{k}}{s} G(s)=V_{k} \frac{a \omega}{2 \sqrt{\xi^{2}-1}}\left(\frac{1}{s+\left(\xi-\sqrt{\xi^{2}-1}\right) \omega}-\frac{1}{s+\left(\xi+\sqrt{\left.\xi^{2}-1\right)} \omega\right.}\right)
$$

Time domain $\mathrm{v}(\mathrm{t})$ :

$$
v(t)=V_{k} \frac{a \omega}{2 \sqrt{\xi^{2}-1}}\left(e^{-\left(\xi-\sqrt{\xi^{2}-1}\right) \omega t}-e^{-\left(\xi+\sqrt{\xi^{2}-1}\right) \omega t}\right)
$$

From expression of 、 can be known, 、 are positive real number,With the t increase of the type will tend to zero:

Most of the time is not concerned about how the crosstalk waveform, and only concerned about whether the crosstalk will affect the normal work of other devices. Therefore, it is very important to estimate the crosstalk peak when usually estimate crosstalk. The above simulation formulas are derived to obtain a simple form of peak value estimation.

Maximum crosstalk value calculated by (5): $\operatorname{mark} \mathrm{t}^{\prime}=\omega \mathrm{t}$, and get the $\mathrm{t}^{\prime}$ derivative:

$$
\begin{aligned}
& v^{\prime}\left(t^{\prime}\right)= 0 \Rightarrow\left(\xi-\sqrt{\xi^{2}-1}\right) e^{-\left(\xi-\sqrt{\xi^{2}-1}\right) t^{\prime}}=\left(\xi+\sqrt{\xi^{2}-1}\right) e^{-\left(\xi+\sqrt{\xi^{2}-1}\right) t^{\prime}} \\
& t^{\prime}=\frac{1}{2 \sqrt{\xi^{2}-1}} \log \left(\frac{\xi+\sqrt{\xi^{2}-1}}{\xi-\sqrt{\xi^{2}-1}}\right)=\frac{1}{\sqrt{\xi^{2}-1}} \log \left(\xi+\sqrt{\xi^{2}-1}\right) \\
& V_{\max } / V_{k}=\frac{a \omega}{2 \sqrt{\xi^{2}-1}}\left[\left(\frac{\xi-\sqrt{\xi^{2}-1}}{\xi+\sqrt{\xi^{2}-1}}\right)^{\left(\xi-\sqrt{\xi^{2}-1}\right) / 2 \sqrt{\xi^{2}-1}}-\left(\frac{\xi-\sqrt{\xi^{2}-1}}{\xi+\sqrt{\xi^{2}-1}}\right)\right. \\
&\left.=\frac{a \omega}{2 \sqrt{\xi^{2}-1}}\left(\xi-\sqrt{\xi^{2}-1}\right)^{\left(\xi / \sqrt{\xi^{2}-1}\right) / 2 \sqrt{\xi^{2}-1}}\left(2 \sqrt{\xi^{2}-1}\right)=a \omega\left(\xi-\sqrt{\xi^{2}-1}\right)^{\xi / \sqrt{\xi^{2}-1}}\right] \\
&=\frac{a \omega}{2 \xi} 2 \xi\left(\xi-\sqrt{\xi^{2}-1}\right)^{\xi / \sqrt{\xi^{2}-1}}
\end{aligned}
$$

Be aware $\frac{a \omega}{2 \xi}=\frac{m_{1}^{2}}{-m_{2}}$, and mark $f(\xi)=2 \xi\left(\xi-\sqrt{\xi^{2}-1}\right)^{\xi / \sqrt{\xi^{2}-1}}$,rewrite the formula of the crosstalk peaks to:

$$
V_{\max } / V_{k}=-m_{1}^{2} / m_{2} \cdot f(\xi)
$$

In the derivation process, need to have $\xi>1$, but (6) for $\xi>0$ were established.

On Figure 3 and Figure 4 ,the solid line shows the simulation results of this paper,the dotted line represents the simulated results of Spice: 


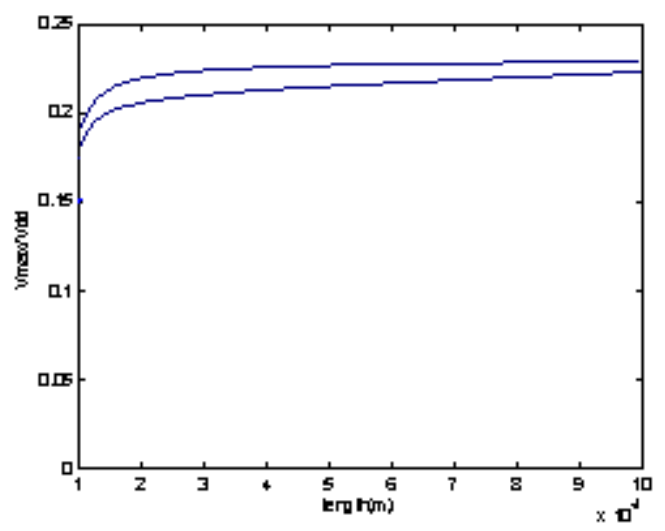

Fig. 3 Relationship between crosstalk peak and interconnection length

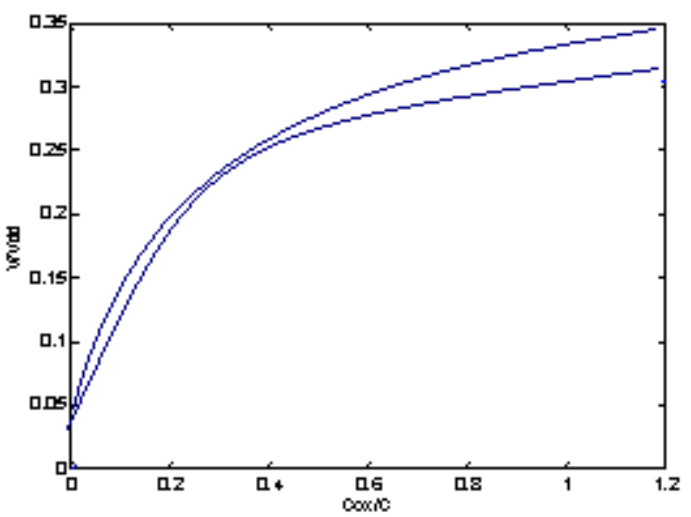

Fig. 4 peak crosstalk estimation under long line conditions

Figure 3 shows the simulation results obtained by (6) under the condition of M $1.1 \mathrm{~m}$ wide, $1 \mathrm{~mm}$ $1.5 \mathrm{~cm}$ long interconnect line under the condition of 0.17 (Drive resistance and load capacitance are corresponding to 100 times the minimum size of the device, the coupling capacitance is $0.08 \mathrm{fF} / \mu$ $\mathrm{m})$. The results of Spice simulation are also given in this paper.From the test data, it is found that the result of the estimation formula is always less than the Spice simulation result.The deviation between the estimated formula and the Spice simulation results is increased with the increase of the length of the interconnect line,it is maintained at about $7 \%$.

In order to investigate the accuracy of the formula (6), it is necessary to analyze the long line situation. When the interconnection line is a long line, $\mathrm{RS}<<\mathrm{R}, \mathrm{CL}<<\mathrm{C}$, the driving resistance and load capacitance can be substituted by zero.At this time, the interconnect length l only affect the time scale of the crosstalk response,has no relation with the crosstalk peak value.Furthermore, it is found that the crosstalk resistance of $\mathrm{R}$ is not related to the peak value of crosstalk. In the long line condition, the peak value of crosstalk is a function of the coupling capacitance Cox and the ratio of to ground capacitance C.Figure 4 shows the comparison between the results of the estimation formula (6) and simulation results of Spice in the long line,the estimated value of the formula (6) is always smaller than the results of the Spice simulation 5\% 7\%,to use the correction formula can be used:

$$
V_{\max } / V_{k}=-K m_{1}^{2} / m_{2} \cdot f(\xi)
$$

Which $\mathrm{K}$ can take 1.35, so formula(7) compared with the results of Spice simulation, the error is less than $3.2 \%$.

Can be drawn on the following properties of $\mathrm{f}: 1) \mathrm{f}$ is a monotone increasing function of $\xi ; 2) \mathrm{f}$ $(0)=0, f(1)=2 / e$, and $\xi$ tends to infinity, $f$ tends to 1.Figure 5 shows the graph of the function $\mathrm{f}$.As can be seen in the $\xi<1$, the rising speed of function $\mathrm{f}$ is fast, and in $\xi>1$, the function $\mathrm{f}$ increased slowly.In the range of $\mathrm{Vmax} / \mathrm{Vk}=0.34 \sim 0.47$ is the most concerned about the peak value of the crosstalk estimation, in practice there is usually a C + CL >Cox, which has: 
$\xi=\frac{1}{2} \sqrt{\frac{m_{2}^{2}}{m_{2}^{2}-m_{3} m_{1}}} \approx 1$

Is there:

$$
\begin{aligned}
V_{\max } / V_{k} & \approx K \frac{4 m_{1}^{2}}{-3 m_{2}} \\
= & \frac{0.3^{*} K\left(R_{S}+\frac{1}{2} R\right)^{2} C_{o x}}{\left(R_{S}^{2}+\frac{5}{6} R_{S} R+\frac{4}{23} R^{2}\right)\left(3 C+\frac{3}{2} C_{o x}\right)+\left(3 R_{S}^{2}+\frac{5}{2} R_{S} R+\frac{5}{6} R^{2}\right) C_{L}}
\end{aligned}
$$

This is because $\xi>1$, the function $\mathrm{f}$ increased slowly. The approximation result used in this estimation formula does not give the formula (8) a too low peak estimate.

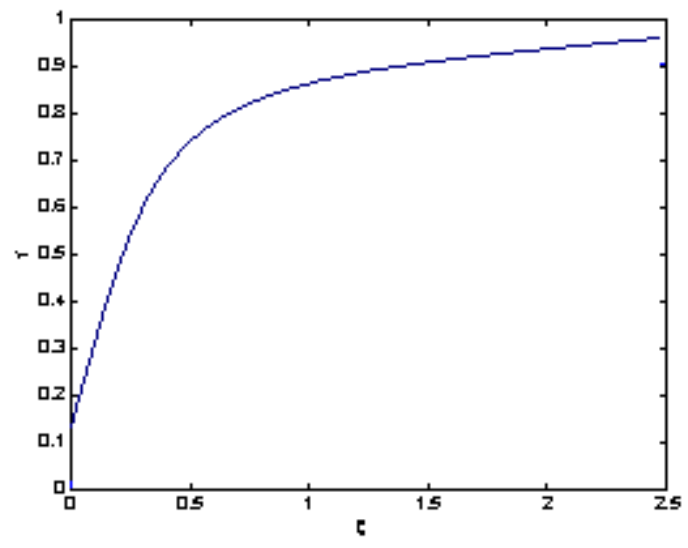

Figure 5 function $\mathrm{f}$ image

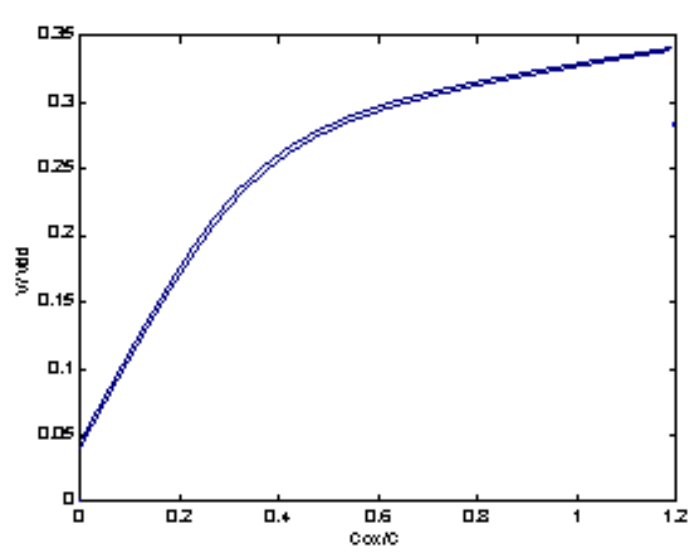

Figure 6 formula(6)and formula(8)comparison

In order to obtain the approximate formula of $V_{\max } / V_{k}$ expressed by S-domain coefficient mi, Can also be directly used $\mathrm{S}$ domain coefficient mi with waveform $\mathrm{v}(\mathrm{t})$ relationship.It is noted that the single step response to the single peak response function of a triangular wave,there have:

$$
V_{\max }=\frac{\int_{0}^{\infty} v(t) d t}{T_{\text {eff }}}=\frac{m_{1}}{T_{\text {eff }}}
$$

$T_{\text {eff }}$ is the $\mathrm{v}(\mathrm{t})$ to achieve the maximum value of $\mathrm{t}, \mathrm{v}(\mathrm{t})$ as a weighted function on the $\mathrm{t}$, the same as $T_{\text {eff }}$ :

$$
T_{\text {eff }}=\frac{\int_{0}^{\infty} t v(t) d t}{\int_{0}^{\infty} v(t) d t}=\frac{-m_{2}}{m_{1}} \therefore \quad V_{\max }=\frac{m_{1}}{T_{\text {eff }}}=\frac{m_{1}^{2}}{-m_{2}}
$$

The input is not a unit step response, $V_{\max } / V_{k}$ remains the same,that is, the formula for the $V_{\max }$. The above is based on the formula of triangle wave compared to the case of the waveform is $\mathrm{v}(\mathrm{t})=t e^{-t}$ difference a constant factor 2/e (about 0.75), it can be adjusted to approximate formula (8), of course, the above formula (8) is strictly derived.

When the interconnection line is a long line, $\mathrm{RS}<<\mathrm{R}, \mathrm{CL}<<\mathrm{C}$,formula (8) simplified as:

$$
V_{\max } / V_{k}=K \frac{7.5 C_{o x}}{16 C+12.6 C_{o x}}
$$

Thus in a given $V_{\max } / V_{k}$ conditions, obtain the corresponding $C_{o x} / C$. Figure 6 shows the comparison between the results of the $K=1$ and the formula (6) when the is taken in the long term. In addition, it can be seen from formula (8) that the peak value of crosstalk is the monotonic 
decreasing function of driving resistance RS and load capacitance CL, which is consistent with the measured data.

\section{Conclusion}

In this paper put forward a complete set of approximate crosstalk estimation model to estimate the model, the model get the CMOS circuit a linear hypothesis, and to use mixed-model with interconnect segment.In the crosstalk estimation model, the coefficients of the low order S domain are obtained, and the approximate estimation formula based on these coefficients is derived, the factors of inductance effect are taken into account, and a new formula is given. The model presented in this paper has a concise expression, compared with the simulation results given by Spice, they can give accurate estimates. Moreover, it can be expected that these models can be implemented simply in the existing CAD model, providing services for high-level synthesis and design.

\section{Acknowledgements}

The authors acknowledge the College Scientific Research Plan Project of Xinjiang Uyghur Autonomous Region (Grant: XJEDU2013I11).

\section{References}

[1] T. Salkerai, "Closed-form expressions for interconnection delay, coupling and crosstalk in VLSI’s”, IEEE Trans. Electron Device, vol. 40, pp. 118-124, Jan. 1993.

[2] Y. Eo, W. R. Eisenstadt, J. Y. Jeong and O. Kwon, "A new on-chip interconnect crosstalk model and experimental verification for CMOS VLSI circuit design”, IEEE Trans. Electron Device, vol. 47, pp. 129-140, Jan. 2000.

[3] J. A. Davis and J. D. Meindl, "Compact distributed RLC models for multilevel interconnect networks”, in Proc. IEEE Symp. VLSI Circuits, pp. 167-168, Jun. 1999.

[4] M. Becer; I. N. Hajj,An analytical model for delay and crosstalk estimation with application to decoupling ,Proceedings IEEE 2000 First International Symposium on Quality Electronic Design (Cat. No. PR00525).

[5] Vobulapuram Ramesh Kumar; Brajesh Kumar Kaushik; Amalendu Patnaik,An Accurate FDTD Model for Crosstalk Analysis of CMOS-Gate-Driven Coupled RLC Interconnects,IEEE Transactions on Electromagnetic Compatibility, Year: 2014.

[6] V. Maheshwari; K. Khare; S. K. Jha; R. Kar; D. Mandal,An analytical crosstalk and delay model for VLSI RLC coupled interconnects,2013 3rd IEEE International Advance Computing Conference (IACC), Year: 2013.

[7] Chandra Sekhar Paidimarry; B Pradeep Kumar; Srinivas Katkoori, A novel approach to crosstalk noise analysis in CMOS inverter driven coupled RLC interconnects,2013 Annual IEEE India Conference (INDICON), Year: 2013.

[8] Ramesh Kumar V.; Kaushik B. K.; Patnaik A,Dynamic crosstalk analysis of CMOS driven RLC interconnects using FDTD method,2013 USNC-URSI Radio Science Meeting (Joint with AP-S Symposium), Year: 2013.

[9] V. Maheshwari, A. Bansal, S.K. Chhotray, et. al., Crosstalk Aware Bandwidth Modelling for VLSI RC Global Interconnects using 2- $\pi$ Mode, Procedia Technology, Volume 6, 2012. 